\title{
Reactive astrocytes augment hippocampal inhibitory tone via GABA transporter-3/4 to facilitate synaptic balance in Alzheimer's disease
}

\author{
Yousif Aldabbagh ${ }^{1}$, Anam Islam ${ }^{1}$, Weicong Zhang ${ }^{1}$, Paul Whiting ${ }^{2}$, and Afia Ali ${ }^{3}$ \\ ${ }^{1}$ UCL School of Pharmacy \\ ${ }^{2}$ UCL Institute of Neurology \\ ${ }^{3}$ University College London School of Pharmacy
}

July 13, 2021

\begin{abstract}
Background and Purpose: Cognitive decline is a major symptom in Alzheimer's disease (AD), which is closely associated with synaptic excitatory-inhibitory imbalance. Here, we investigated whether astrocytic mechanisms involving the astrocyte-specific GABA transporter 3/4 (GAT3/4) play a role in altering the synaptic balance in AD and whether these mechanisms correlate with presynaptic cannabinoid type-1 receptors (CB1-Rs). Experimental approach: Using the APPNL-F/NL-F knock-in mouse model of $\mathrm{AD}$, aged-matched to wild-type mice, we performed in vitro electrophysiological whole-cell recordings combined with immunohistochemistry in the CA1 and dentate gyrus (DG) regions of the hippocampus. Comparative neuroanatomy experiments were also performed in post-mortem brain tissue from human AD patients, age-matched to healthy controls. Results: We observed a higher expression of GABA content and GAT3/4 co-localised with reactive astrocytes, which enhanced tonic inhibition in the CA1, and DG of APPNL-F/NL-F mice compared to the age-matched wild-type animals. Blocking GAT3/4 - associated tonic inhibition in APPNL-F/NL-F mice resulted in an enhanced frequency of synaptic excitation, suggesting a presynaptic mechanism. These data also correlated with an up-regulation of CB1-Rs in astrocytes and cholecystokinin (CCK)containing interneurons, which also enhanced tonic inhibition in the AD model, but did not affect GAT3/4 -associated tonic inhibition. The neuroanatomical results were mirrored in post-mortem tissue of AD patients. Conclusions: Our data suggest that reactive astrocytes lead to augmented tonic inhibition in the hippocampus, which probably plays an important presynaptic compensatory role in attempting to restore AD-associated neuronal hyperactivity. Therefore, reducing tonic inhibition through GAT3/4 may not be a good therapeutic strategy for AD.
\end{abstract}

\section{Hosted file}

Aldabbagh et al GAT3_BJP_FullManuscript_Submission Ready.docx available at https://authorea. com/users/425396/articles/530228-reactive-astrocytes-augment-hippocampal-inhibitorytone-via-gaba-transporter-3-4-to-facilitate-synaptic-balance-in-alzheimer-s-disease 

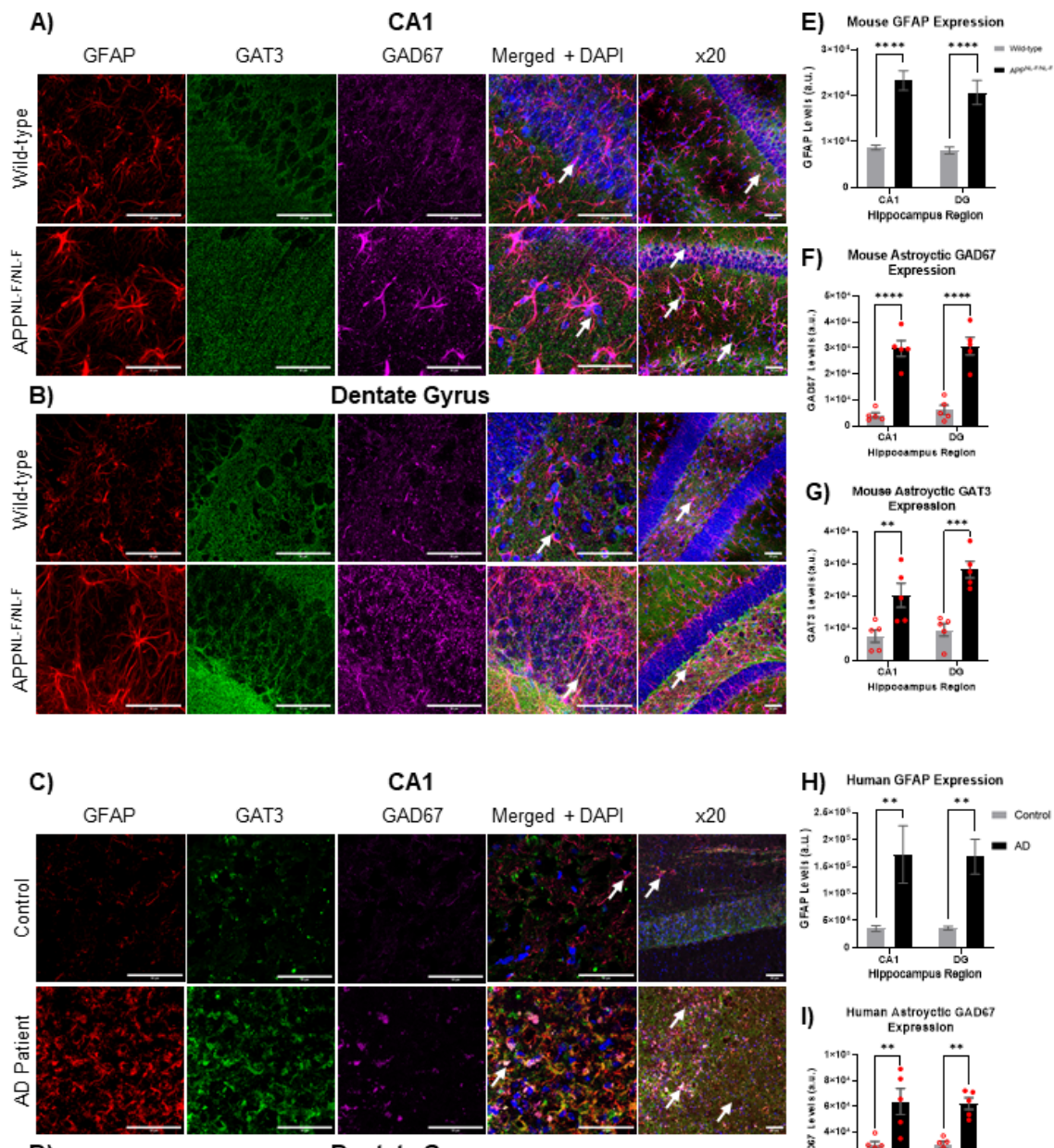

H) Human GFAP Expression
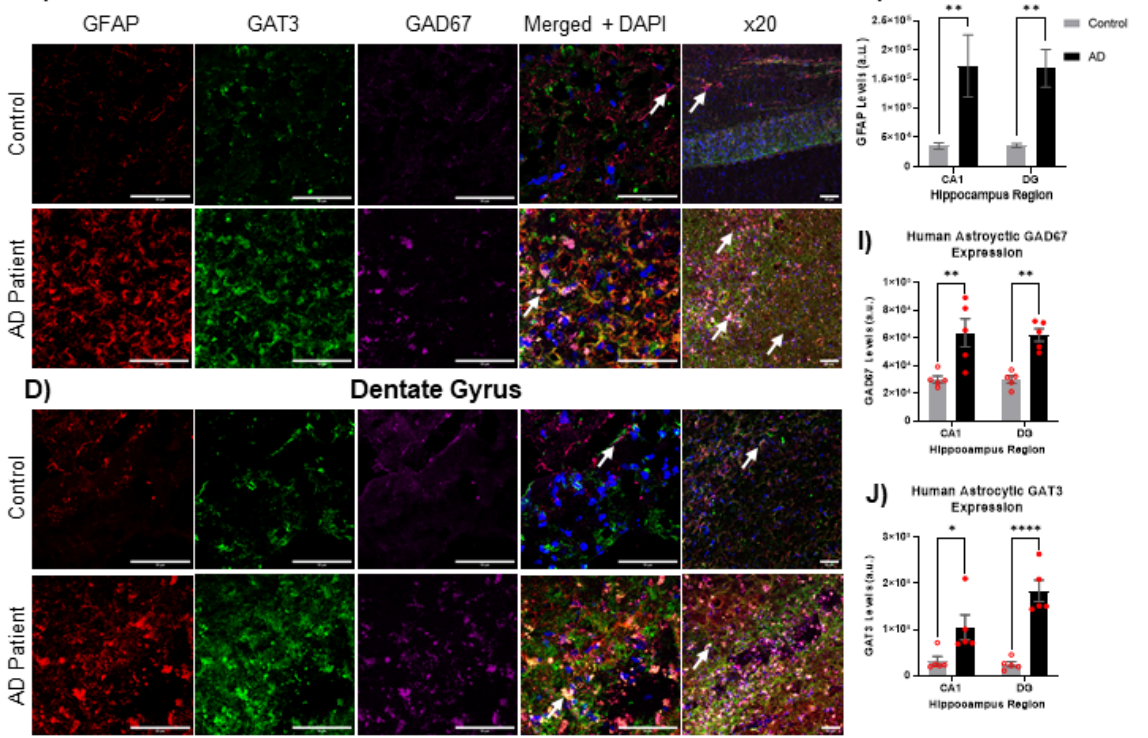

J) 


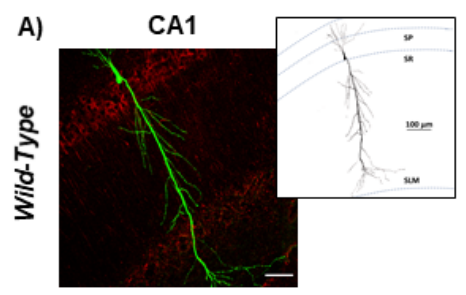

C)
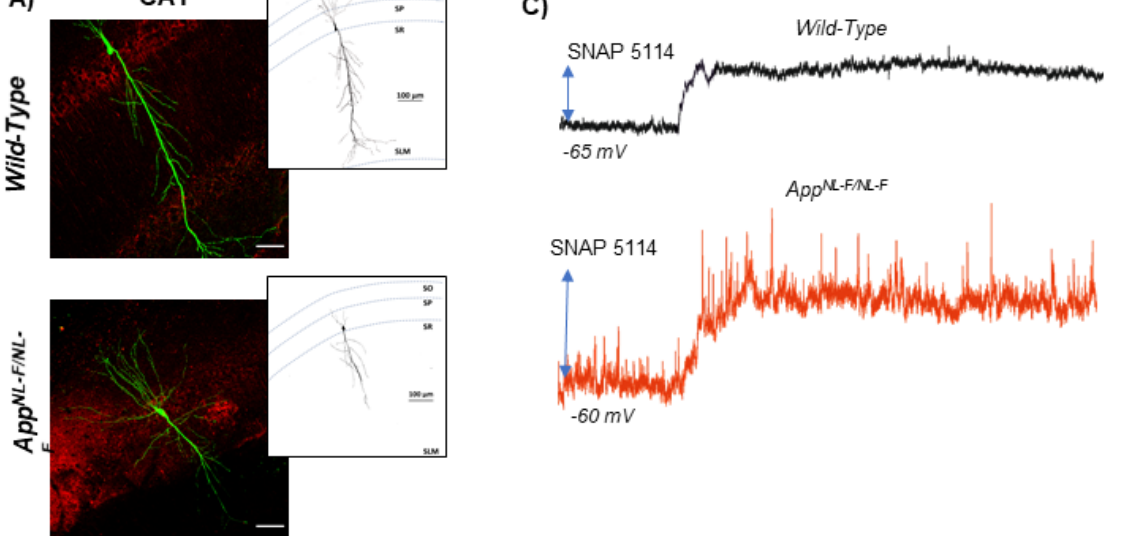

B) Dentate Gyrus

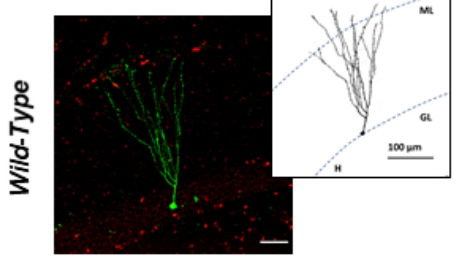

D)

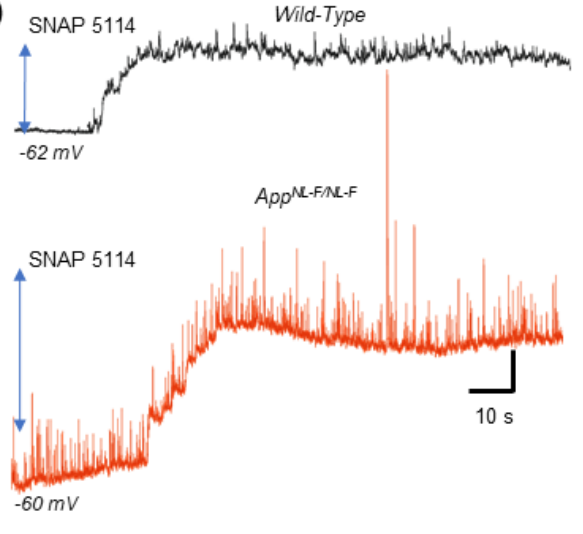

E)

- Control

F)

G)

Q Wild-Type
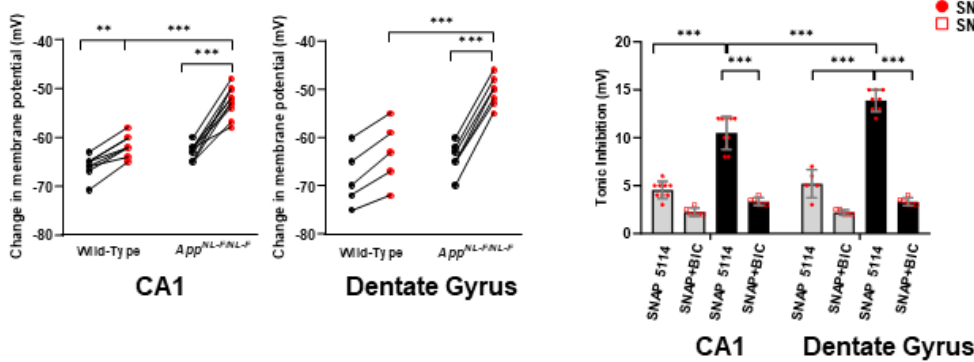

$\square$ SNAP + BIC 

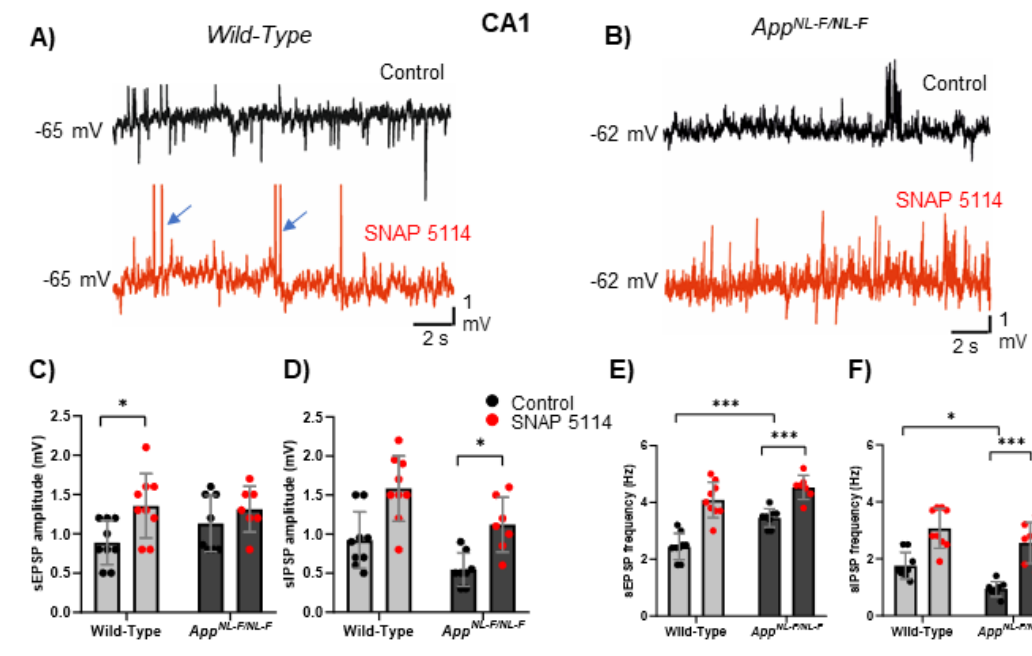

D)

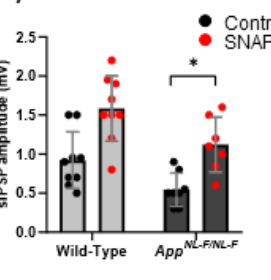

E)

F)
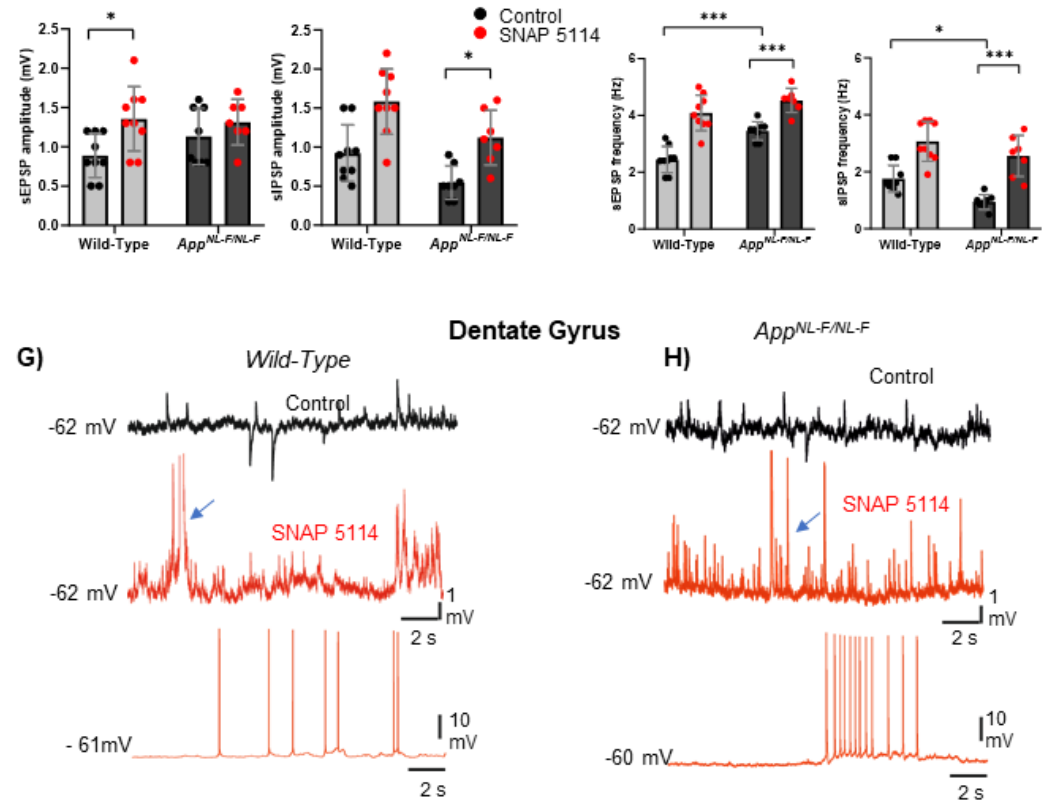

Dentate Gyrus $\quad$ H) $\quad A p p^{N L-F / N L-F}$
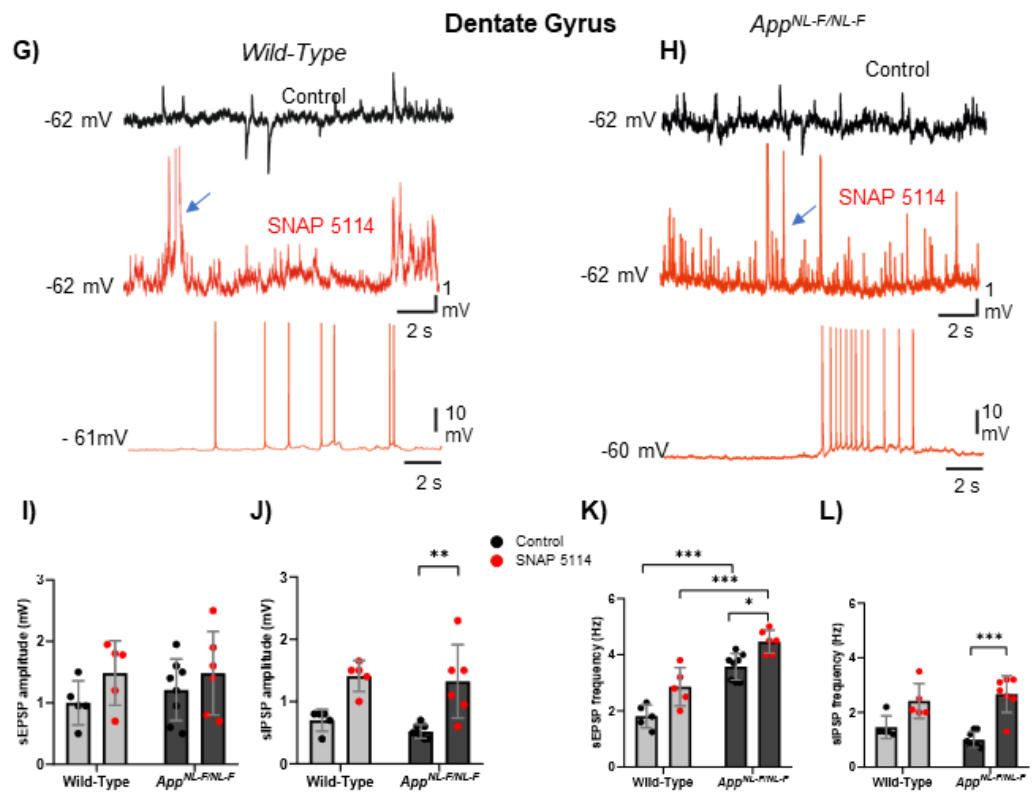

J)

K)

L)

$\stackrel{* *}{\bullet}: \begin{gathered}\text { Control } \\ \text { SNAP 5 514 }\end{gathered}$
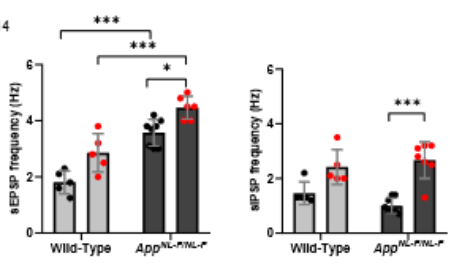

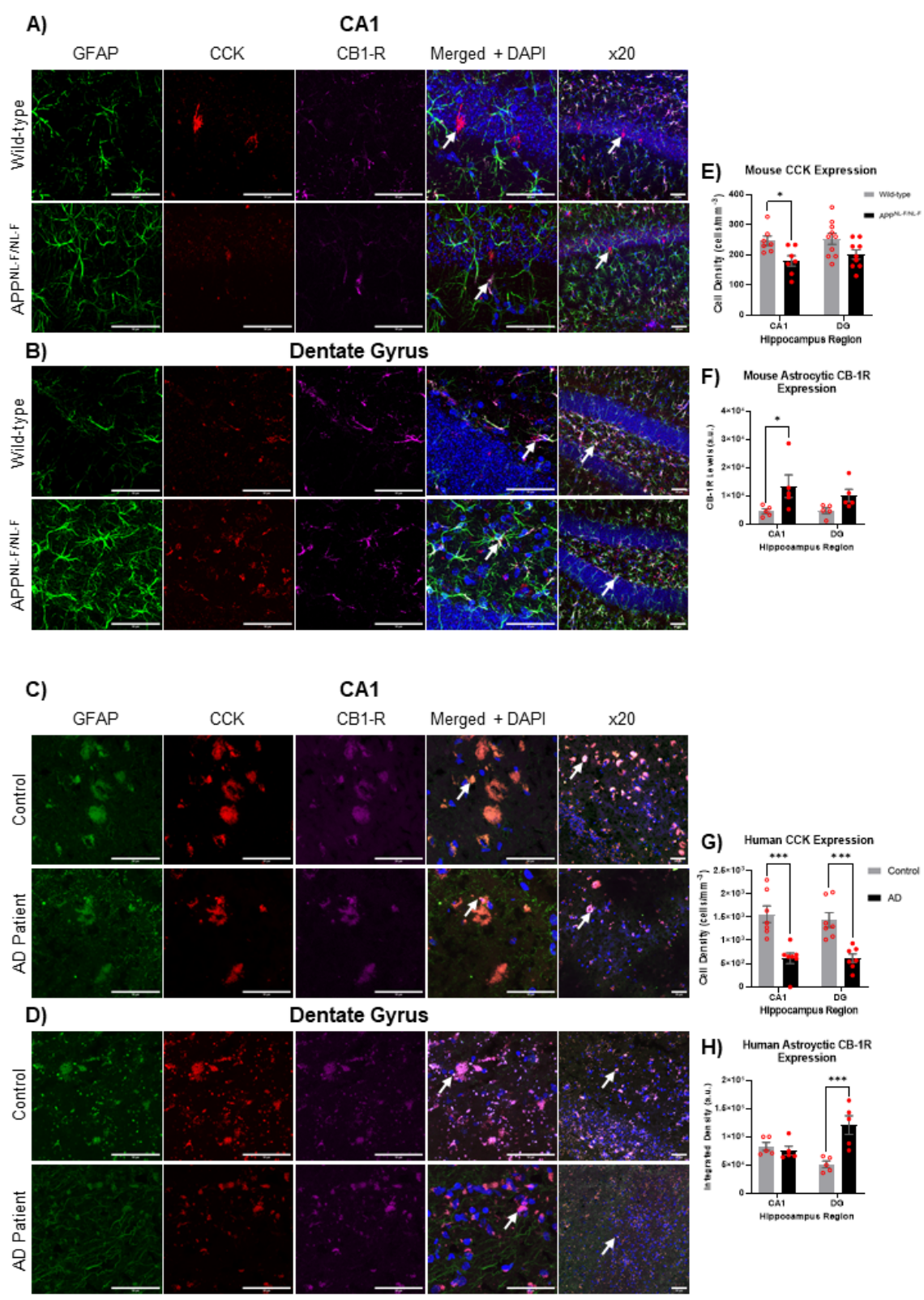


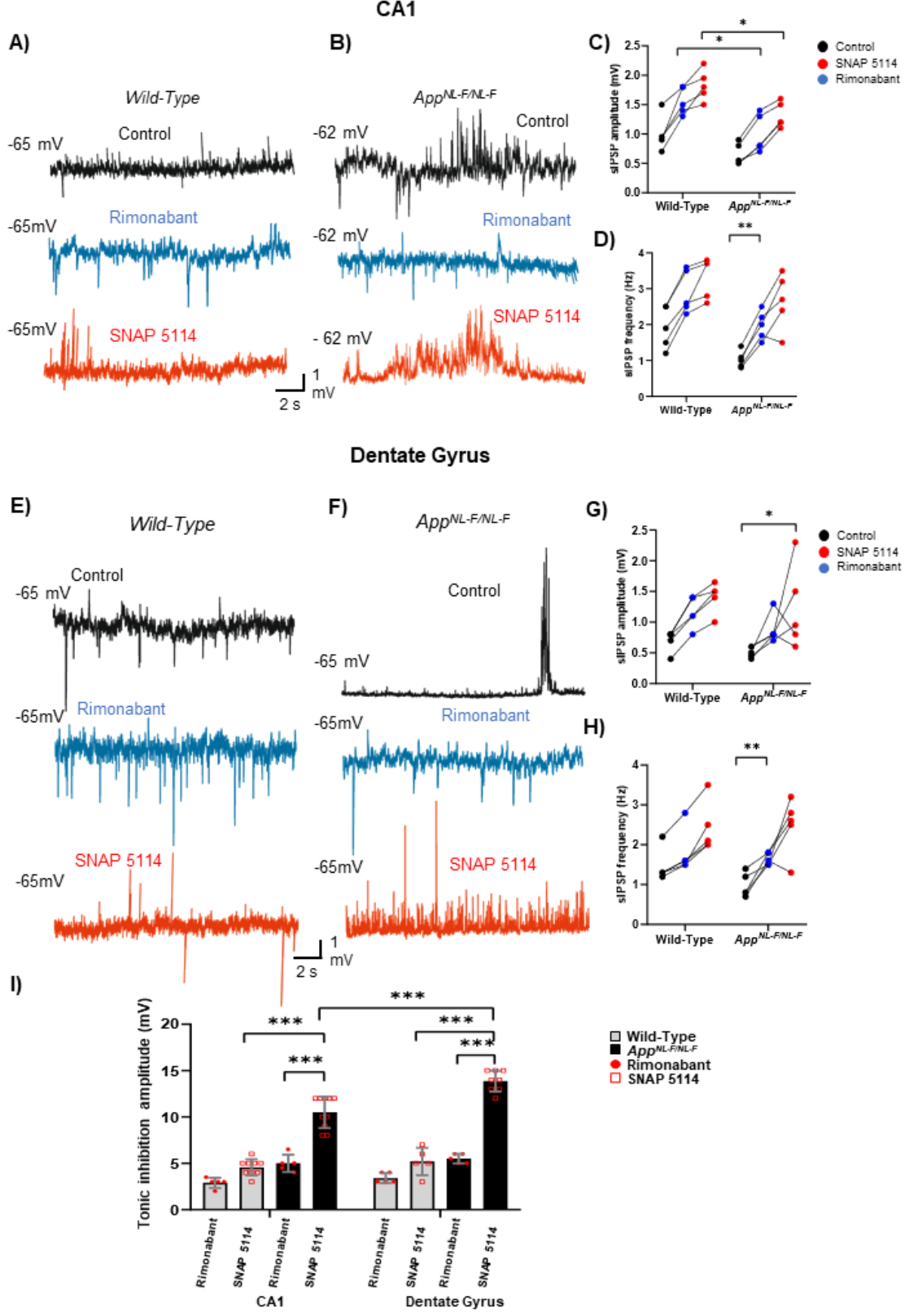




\section{Supplementary Figure 1}

A)

Mouse GFAP/GAD67 Colocalisation

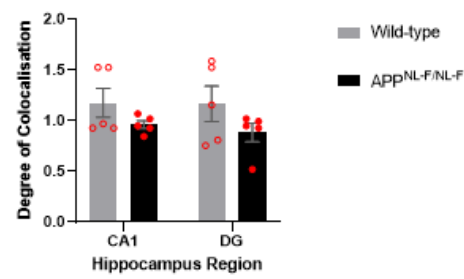

C) Mouse GFAP/GAT3

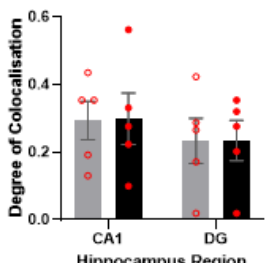

E) Mouse GFAP/CB1-R Colocalisation

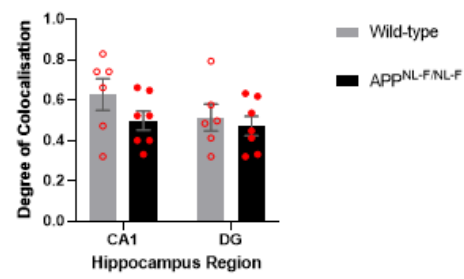

B) Human GFAP/GAD67 Colocalisation

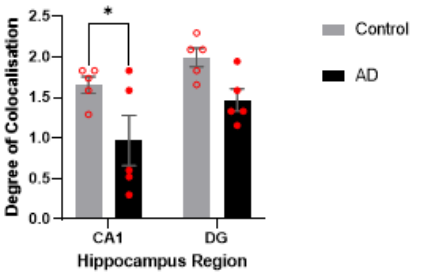

D) Human GFAP/GAT3

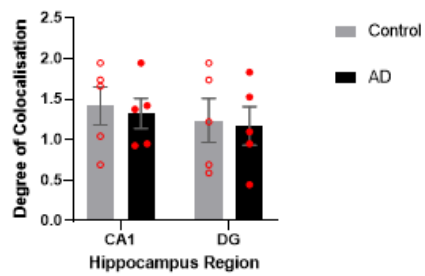

F) Human GFAP/CB1-R

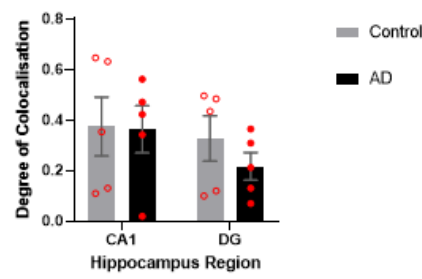

